\title{
Single-phase AC rectifier with high power factor
}

\author{
Vladlen Ivanov \\ Novosibirsk State Technical University \\ Novosibirsk, Russia \\ NSTU \\ ivanov.etk@yandex.ru \\ Pavel Lisitsyn \\ Novosibirsk State Technical University \\ Novosibirsk, Russia \\ NSTU \\ nik-nokia@mail.ru
}

\author{
Sergei Myatez \\ Novosibirsk State Technical University \\ Novosibirsk, Russia \\ NSTU \\ serg_y_7578@mail.ru
}

\author{
Andrei Kapustin \\ Novosibirsk State Technical University \\ Novosibirsk, Russia \\ NSTU \\ kapusta_nsk@mail.ru
}

\author{
Irina Alekseeva \\ Novosibirsk State Technical University \\ Novosibirsk, Russia \\ NSTU \\ alekseeva1201@mail.ru
}

\begin{abstract}
This paper analyzes the known methods of ensuring electromagnetic compatibility of average single-phase AC consumers having semiconductor rectifiers, thus having nonlinear I-V curves, with a power supply network. The traditional method of single-phase AC-DC conversion for domestic needs and industrial automation is based on the use of common bridge rectifiers with a capacitive load having low power characteristics that are to be improved by additional correcting devices. Reconsideration of the way of interaction between a rectifier and its smoothing capacitor used as an energy accumulator gives additional possibilities for increasing power characteristics of power sources with such rectifiers and improving electromagnetic compatibility with a single-phase $\mathrm{AC}$ supply network.
\end{abstract}

Keywords - Power factor, electromagnetic compatibility, single-phase AC rectifier, distortion coefficient, efficiency.

\section{INTRODUCTION}

Development of semiconductor technology gives new solutions for various domestic and industrial AC power consumers (e.g. automation devices, microprocessor-based devices, frequency converters, energy saving lamps, and others). Such devices represent loads with nonlinear currentvoltage characteristics (I-V curves) that causes a serious worldwide problem of electromagnetic compatibility with existing AC supply networks [1].

These networks were initially designed for simple consumers having conditionally linear I-V curves (e.g. heating devices, electric motors without semiconductor converters, incandescent lamps). Therefore, they were not suitable for currents with considerable harmonic levels (up to $85 \%$ and more) that cause overheating of current-carrying elements, distortions of power supply voltage and other negative effects.
To solve this problem, it is reasonable not only to reconsider design regulations for electrical networks of industrial and domestic consumers, but also to improve ACDC conversion techniques.

\section{METHODS FOR INCREASING THE POWER FACTOR FOR SINGLE-PHASE AC CONSUMERS}

The principle of operation of all known technical solutions for power factor increasing involves impulse stretching for input supply source current in such a manner as to approximate its form and a phase shift to a supply voltage curve. In this case, a consumer will be able to take energy from the network gradually without any overloads and distortions that is similar to the behaviour of an active load having a linear I-V curve.

Technical solutions for power factor increasing can be divided into two groups: filter devices, which suppress undesirable harmonics of input current impulse by impulse stretching, and power factor correctors, which modify (correct) the load character. There is a broad classification of technical solutions for these two groups that represents the principles of their operation (e.g. passive or active filter, lowfrequency or high-frequency PWM corrector). Such variety demonstrates that there is no the best technical solution for power factor increasing. Each variant has its own features and limitations.

For example, in the case of average single-phase AC consumers, having rectifiers, low-pass filters (LPF) connected at the power supply source input (Fig. 1) are widely used in practice. 


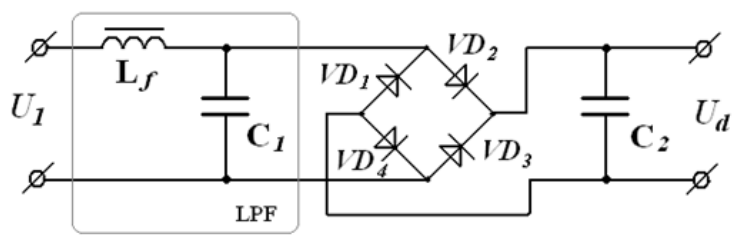

Fig.1. Connection of a low-pass filter at the input of the single-phase AC rectifier

Their efficiency is illustrated in Fig. 2.

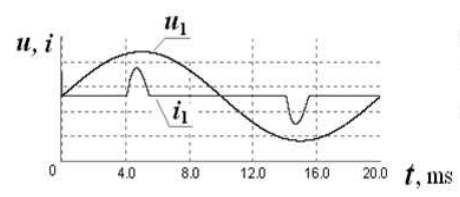

$a$

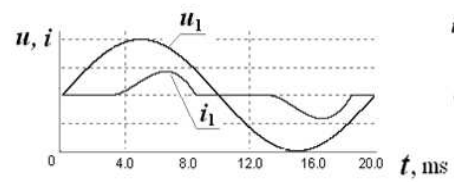

$c$

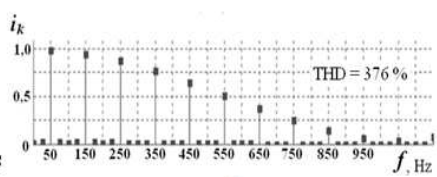

$b$

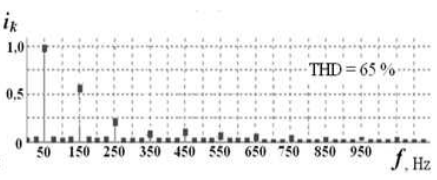

$d$
Fig.2. LPF operation to the input rectifier current - $(\mathrm{a}, \mathrm{c})$ and the current higher harmonic spectrum - $(\mathrm{b}, \mathrm{d})$ depending on the following LPF parameters: for $L_{f}=3 \mu H$ and $C_{l}=0, \ln F-(\mathrm{a}, \mathrm{b})$; and for $L_{f}=3 m H$ and $C_{1}=0,1 \mu F-(\mathrm{c}, \mathrm{d})$

Therefore, the efficiency of low-pass filters is directly influenced by the parameters of their reactive elements. In practice, dimensions and prices for input chokes $L_{f}$, significant elements for filtering, do not allow developing high-performance filter devices to be used for the most of single-phase AC consumers. As a result, this technical solution should be considered as only an additional technique of power factor increasing.

The decrease of reactive element dimensions for power supply sources in technical solutions of power factor increase is possible when using the higher conversion frequency in comparison with the supply network. This capability is provided by high-frequency power factor correctors operated at frequencies of more than tens of $\mathrm{kHz}$. The principle of operation of these correctors is based on the artificial generation of the desired input current curve by switchings of electrical circuits with reactive elements by semiconductor power devices (SPD). Fig. 3 gives a traditional diagram of an active high-frequency power factor corrector as a part of a single-phase rectifier where the reactive element $L_{P F}$ is switched by the transistor $V T$ according to one of the numerous known algorithms [6].

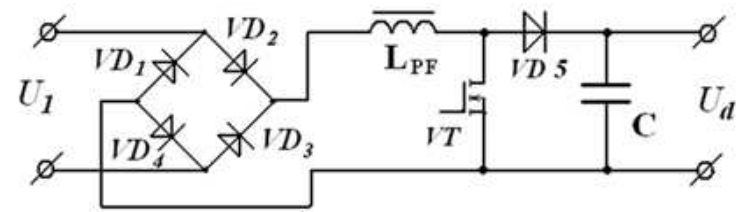

Fig.3. Single-phase rectifier with an active high-frequency power corrector
It should be noted that operation of such technical devices will be effective if the system has sensors of input and output voltage and current, which signals are processed using adaptive algorithms. To realize these algorithms, microcontroller-based devices are needed that provide implementation of various correction algorithms, including stabilization of power supply source parameters.

Operation of properly chosen active correctors may give the desired effect (Fig. 4), but connection of additional microcontrollers with an adaptive algorithm of power factor active correction into a power source of each single-phase AC consumer inevitably increases costs and decreases reliability indexes.

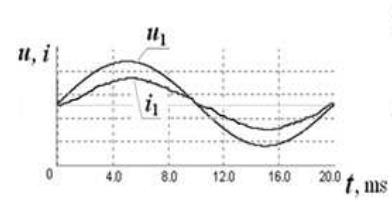

$a$

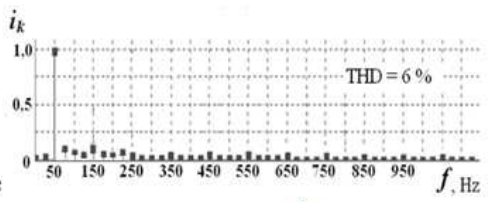

$b$
Fig.4. Influence of the active high-frequency power factor corrector on the rectifier input current (a) and higher harmonic spectrum (b)

It should be noted that technical solutions of power factor increasing, inherently, only eliminate the drawback caused by operation of a common single-phase rectifier with a capacitive load.

\section{IMPROVEMENT OF A SINGLE PHASE RECTIFIER AC AS A PART OF THE POWER SOURCE}

A capacitor, being a compact and inexpensive energy accumulator for smoothing fluctuations during single-phase rectifier operation, is chosen for a reason. It has the highest specific energy parameter (i.e. energy per unit mass) among existing electronic components. For modern capacitors, specific energy reaches $1 \mathrm{~kJ} / \mathrm{kg}$ and higher, while this parameter for inductances is lower by two-three orders of magnitude [7].

For this reason, the switching problem occurring during interaction of two sources of electromotive forces (EMF), an AC power supply network and a capacitor as a temporary EMF source (as is known, capacitor voltage cannot be changed by step), should beaccepted (Fig. 5).
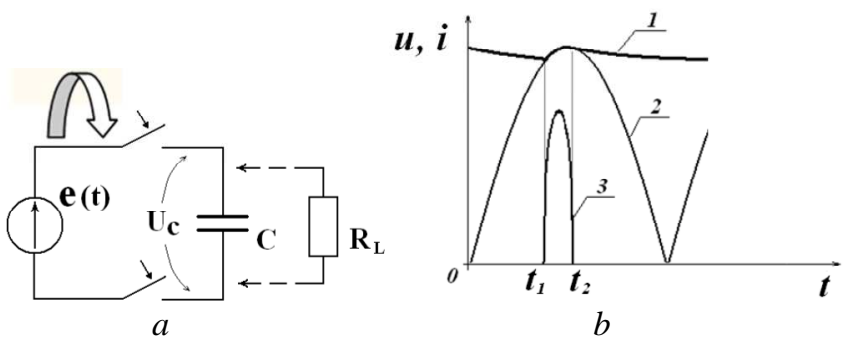

Fig.5. Interaction of a partially charged capacitor and a power supply network: $\mathrm{a}$ - circuit at the moment of switching; $\mathrm{b}$ - curves of voltage and current ( 1 capacitor voltage; 2 - network voltage $e(t) ; 3$ - capacitor charging current)

Under normal conditions, capacitor charging in the rectifier, except voltage drops at semiconductors are realized if $e(t)>U_{C}$. 
Since power sources are designed in such a way that voltage fluctuations at a smoothing capacitor (curve 1 in Fig. $5 b$ ) should not exceed several percents, then capacitor charging is possible within a short time period of about $t_{2}-t_{1} » 5 . .15^{\circ}$.

Therefore, for keeping the capacitor as an energy accumulator in the single-phase rectifier to eliminate fluctuations and for extending a time interval of capacitor charging without any schematic solutions, such as filters and correctors, it is necessary to consider a possibility of deep capacitor discharge under operating conditions.

Taking into account that the peak value of the sinusoidal network voltage is actually overloaded by numerous consumers [1,4] with considerable voltage distortions in different nodes then a power supply source used for charging a deeply discharged capacitor will have a converse effect, that is provide partial or full elimination of such typical distortions.

To verify this situation, operation of the power supply system will be simulated with the distorted peak value of the sinusoidal network voltage. After that, the influence of the considered device connected to the circuit by $S A$ switch will be determined with recording of the change of the voltage form (Fig. 6).

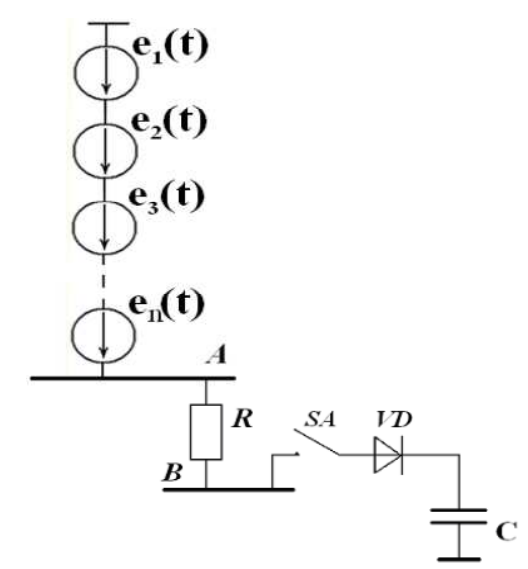

Fig.6. Investigations of compatibility between the device of complete capacitor charging

Simulation results show that the considered device contributes for elimination of voltage distortions in the power supply network. For example, higher harmonic level in the network voltage determined at busbars as $T H D=5,3 \%$ is reduced to the level of $T H D=4,1 \%$ in the connection point of the device.

Therefore, the capability of correction of currents, voltages and powers is designed into the rectifier if cycles of full charge / deep discharge of the capacitor are permitted. If varying the depth of capacitor discharge and the moment of its connection to the network by SPD, it is possible to correct the regime of energy consumption in the range from $0^{\circ}$ to $90^{\circ}$ for a positive half-wave and in the range from $180^{\circ}$ to $270^{\circ}$ for a negative half-wave by developing a circuit with inverse SPD connection.
For separating processes of full charges and deep discharges of the capacitor and the process of load power supplying, it is necessary to use the system of two capacitors with their static rotation.

The first capacitor consumes energy from the network, while the second capacitor releases stored energy to the DC load side.

Such system does not solve the problem of energy storage from the AC network side in the ranges from $90^{\circ}$ to $180^{\circ}$ and from $270^{\circ}$ to $360^{\circ}$. Actually, these ranges are almost closed from energy storage by simple rectifiers operated to the capacitive load. The network voltage gradually decreases from the peak value to zero within these ranges that conflicts with the condition of normal capacitor charging. To solve this problem, the principle implied in the device[2] of passive power factor correction (Fig. 7) can be used.

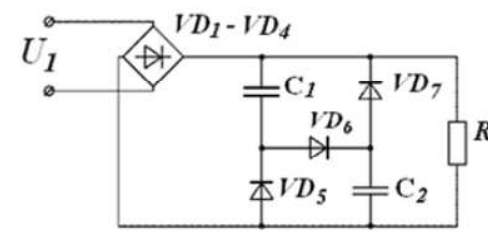

$a$

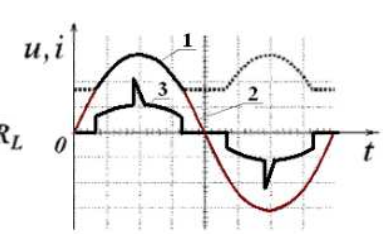

$b$
Fig.7. Rectifier with two-level capacitor switching of the power factor corrector: $a$ - circuit diagram; $b$ - oscillograms of currents and voltages ( 1 load voltage, 2 - network voltage, 3 - input network current)

In this system, capacitors $C_{1}$ and $C_{2}$ are charged from the network through the diode $V D_{6}$, being connected in series, and discharged to the load $R_{L}$ through diodes $V D_{5}$ and $V D_{7}$, being connected in parallel.

In spite of the fact that such rearrangement of capacitors due to diode switching involves considerable (about 50\%) voltage fluctuations at the load side, the device consumes current from the network within broad intervals: from $30^{\circ}$ to $150^{\circ}$ for the positive wave, and from $210^{\circ}$ to $330^{\circ}$ for the negative wave of the network voltage.

Based on the principle of capacitor variation with dynamic changes in capacitor connections and their combinations by controlled SPD switchings, it is possible to ensure capacitor charging even within time intervals of decreasing network voltage.

This switching sequence represents positional multi-stage charging for separate capacitor sections. Moments of thyristor triggering into the conducting state can be determined by comparing voltages of separate capacitor sections (Fig. 8) with current instantaneous value of the supply network voltage that is decreasing in the interval from $90^{\circ}$ to $180^{\circ}$. 


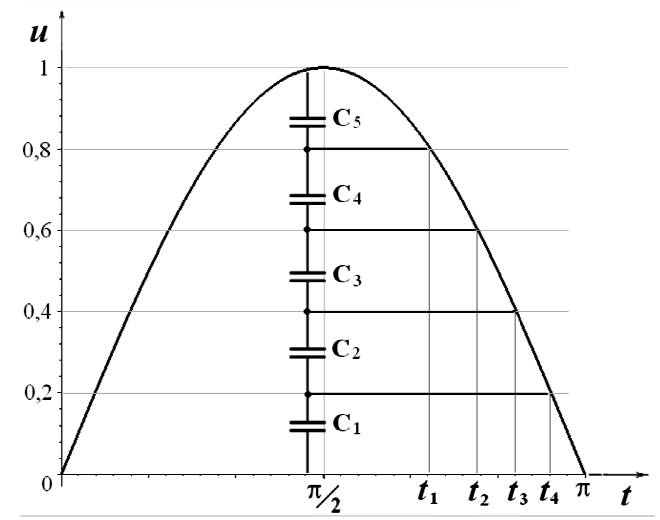

Fig.8. Overlapping of the circuit diagram of capacitors and the waveform of the supply network voltage

For example, the device has five capacitor sections to be switched by SPD. Let us assume that the supply network voltage has the sinusoidal waveform with the relative amplitude $\boldsymbol{u}^{*}$ and the frequency $\boldsymbol{f}=50 \mathrm{~Hz}$. Capacitor voltages are equal because of their equal capacitances. Capacitors are previously charged in the first part of the positive half-cycle (interval of $0-\pi / 2$ ). Then, the moments of thyristor triggering into the conducting state will be known in advance:

$$
t=\frac{\pi-\arcsin \left(u^{*}\right)}{2 \pi \cdot f} .
$$

Therefore, the first thyristor should be triggered into the conducting state in advance at the moment of $t_{1}$ when the supply network voltage is

$$
U_{C 1}+U_{C 2}+U_{C 3}+U_{C 4}=0.8 \leq u\left(t_{1}\right) .
$$

In a similar way, conditions of triggering into the conducting state for the second and other thyristors are:

$$
\begin{gathered}
U_{C 1}+U_{C 2}+U_{C 3}=0.6 \leq u\left(t_{2}\right), \\
U_{C 1}+U_{C 2}=0.4 \leq u\left(t_{3}\right), \\
U_{C 1}=0.2 \leq u\left(t_{4}\right) .
\end{gathered}
$$

Since the moments of advance thyristor triggering into the conducting state are regulated by a control system, the following conditions can be obtained: the energy stored by capacitors from the network in the first part of the positive half-cycle will be equal to the energy stored by capacitors from the network in the second part of the positive half-cycle. Therefore, it is possible to correct intensity of energy consumption by the power supply source that is similar to operation of active power factor correctors.

Electric energy consumption by the proposed power source is realized in a similar way in both parts of the negative halfcycle from $180^{\circ}$ to $360^{\circ}$. In this case, the second subsystem of capacitors and thyristors should be considered. Thyristors should be connected in such way to operate with the negative half-wave of the voltage.

While the second subsystem of the power source consumes energy from the AC network, the first subsystem should release energy stored by capacitors to a DC consumer, thus implementing the principle given in Fig. 8 by controlled SPD switchings.

Fig. 9 illustrates the possible variant of the circuit design for the proposed device.

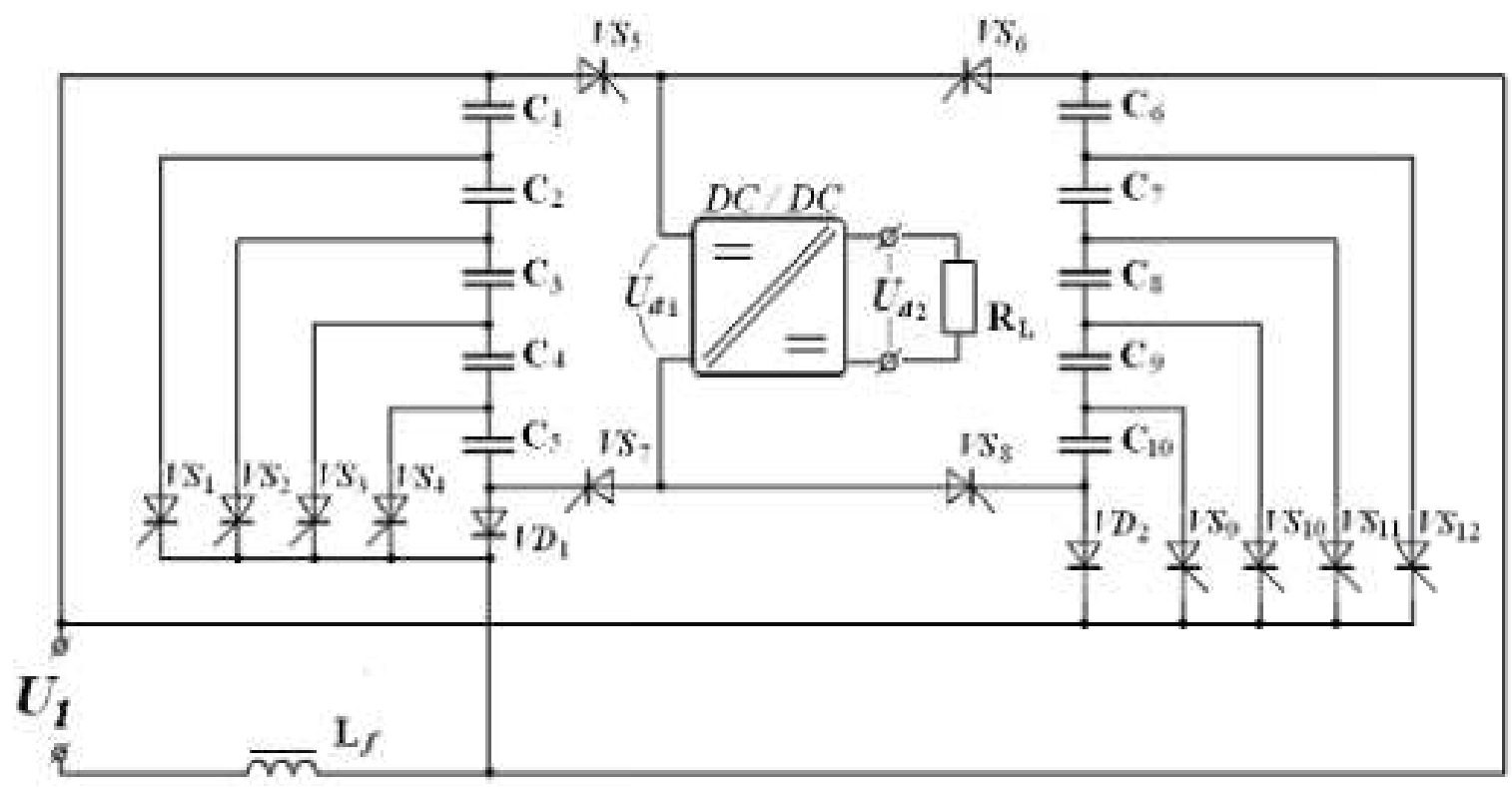

Fig.9. Circuit design of the power source providing the rotation principle and five-stage capacitor charging 


\section{CONCLUSIONS}

The idea of improving energy characteristics of singlephase rectifiers with capacitive energy storages by changing the processes of electric energy accumulation and release without any additional correcting devices is proposed.

Based on the single-phase rectifier with multi-stage capacitor charging, the compact power-supply source is proposed. Parameters of this device are close to the parameters of power sources with active power factor correctors.

It was shown that the proposed principle of rotation of charged capacitors of the single-phase rectifier as part of the power supply source provides galvanic isolation of a DC consumer from the supply network. In this case, energy transfer is realized only by installed capacitors.

\section{REFERENCES}

[1] M.S. Witherden, R. Rayudu, R. Rigo-Mariani, "The influence of nonlinear loads on the power quality of the New Zealand low voltage electrical power distribution network," Power Engineering Conference, pp. $102-108,2010$.

[2] S.G. Marushchenko, "The influence of the secondary power source of a LED illuminator on the electrical network," Modern high technologies, No.9, pp. 103-107, 2013.

[3] G.S. Zinovyev, Power electronics, Moscow, Yurait, 2016.

[4] P. Hunter, "Solve Switcher Problems With Power-Factor Correction," Electronic Design, pp. 67-78, February 1992.

[5] V.P. Klimov, "Modern directions of the development of AC power converters," Practical power electronics, No.25, pp. 43-51, 2007.

[6] S. Reznikov, V. Bocharov, Ye. Parfenov, N. Gurenkov, A. Kornilov, "Electric and electromagnetic compatibility of secondary impulse power sources with autonomous AC power supply systems," Power electronics, No.5, pp. 86-89, 2009.

[7] V.A. Aleshkevich, University course of general physics. Electromagnetism, Moscow: Fizmatlit, 2014.

[8] V. V. Ivanov, S. V. Myatez, A. V. Kapustin and I. K. Alekseeva, "Development prospects of single-phase zone rectifiers," The 11 International forum on strategic technology (IFOST 2016), Novosibirsk : NSTU, Pt. 2, pp. 105-107, 2016.

[9] A.V. Plaks, Control systems of electric rolling stock, M.: Training Center for Education in rail transport, 2005, p. 360.

[10] G.S. Zinovev, Bases of the power electronics, Novosibirsk, NSTU, 2003, p. 664.

[11] V. V. Ivanov, S. V. Myatez, E. G. Langeman, N. I. Schurov, "Pulse-width control in ladder structure four-phase rectifier for AC-electromotive," IOP Conf. Series: Materials Science and Engineering, 127(1) 012004. 2016,

[12] B.G. Yuzhakov, Electric drive and converters of rolling stock: Textbook for technical schools and colleges of rail transport, M.: SEI Educational Centre for Education in rail transport, 2007, p. 396.

[13] L.A. Bessonov, Theoretical Foundations of Electrical Engineering, Electrical circuits: Ucheb.dlya university students.-10 Ed. M .: Gardariki, 1987. p. 293.

[14] E. Y. Abramov, A. A. Stang, S. A. Enkudinov, "Transformation of the urban electric transport system when using autonomous energy sources," Advanced Materials Research High technology: research and applications, pp.714-718, 2014.

[15] S.A. Evdokimov, L.G. Evdokimova, "One-phase AC-DC rectifier", Patent of Russia №2398344, 2009.

[16] B. A. Arzhannikov, A. A. Pyshkin, Improving of DC power supply system based on automatic voltage regulation of traction substations, Ekaterinburg: USURT Press, p. 116, 2006.
[17] E. Y. Abramov, "Experimental investigation of energy parameters of urban electric transport traction substations", Proceedings of the Russian higher school academy of science, 3 (32), pp. 33-42, 2016. 